\title{
Estudio comparativo de la adsorción de azul de toluidina y cristal violeta por la biomasa de dos especies del género trichoderma y carbón activado
}

\author{
Rosalba Argumedo-Delira ${ }^{a^{*}}$, Alejandro Alarcón ${ }^{\mathrm{b}}$, Ronald Ferrera-Cerrato ${ }^{\mathrm{b}}$, Ma. Remedios Mendoza- \\ López $^{\mathrm{a}}$, Oscar García-Barrada ${ }^{\mathrm{a}}$ y Jesús Samuel Cruz-Sánchez ${ }^{\mathrm{a}}$.
}

\author{
${ }^{a}$ Unidad de Servicios de Apoyo en Resolución Analítica (SARA), Universidad Veracruzana. Luis Castelazo Ayala s/n Col. Industrial \\ Animas 91190, Xalapa, Veracruz, México. \\ ${ }^{b}$ Área de Microbiología. Postgrado de Edafología. Colegio de Postgraduados. Carretera México-Texcoco Km 36.5, Montecillo 56230 , \\ Estado de México. México. \\ *Email de autor responsable: rargumedo@uv.mx, rosasusana13@hotmail.com
}

Recibido 06-septiembre-2013, Aceptado 18-octubre-2013

\section{Resumen}

El Cristal Violeta (CV) y el Azul de Toluidina (AT) han sido encontrados en ecosistemas terrestres y acuáticos debido a su inapropiada disposición. Para disminuir la contaminación que provocan dichos colorantes al medio ambiente se han buscado alternativas biológicas, dentro de las cuales se encuentra el uso de la biomasa fúngica como agente adsorbente. Considerando lo anterior el presente trabajo determinó la biosorción de la biomasa de dos especies del género Trichoderma frente a CV y AT, y la comparó con la adsorción que presenta el carbón activado ante estos dos colorantes. Los resultados indicaron que el promedio de biosorción de CV por la biomasa de T. viride (96\%) y T. virens (94\%) no fueron tan altos como la del carbón activado (99\%). En contraste, el promedio de adsorción para AT fue mucho menor para la biomasa de $T$. viride $(83 \%)$, T. virens $(84 \%)$ y carbón activado $(93 \%)$ que el que tuvieron ante CV. Los datos experimentales tienen una buena correlación con los modelos de Freundlich, Langmuir y el modelo cinético de pseudo segundo orden. Encontrando una qmax de $10.39 \mathrm{mg} \mathrm{g}^{-1}$ para $T$. viride en el caso de CV y de $21.69 \mathrm{mg} \mathrm{g}^{-1}$ para $T$. virens ante AT.

Palabras clave: Colorante, Trichoderma, biosorción, biomasa, micelio

Keywords: Dye, Trichoderma, biosorpion, biomass, mycelium

\section{Introducción}

Los colorantes son sustancias orgánicas que sirven para dar color a diversos productos comerciales (tales como lana, seda, algodón, lino, rayón, nylon, papel, cosméticos, alimentos y plásticos) de un gran número de industrias y además son compuestos complejos con varios anillos aromáticos de carácter recalcitrante [1,2]. El Cristal Violeta (cloruro de N,N,N',N',N",N"hexametil-p-rosanilina) es ampliamente utilizado como un agente dermatológico, agente de tinción, medicamento veterinario (contra hongos y parásitos) y en la industria textil-papelera [3]. Mientras que el Azul de Toluidina (Sal disódica del ácido-2,2' $-[(9,10$-dihidro4,8-dihidroxi-9,10-dioxo-1,5-antracenedil)

diimino]Bis[5-metil bencensulfónico]) ha sido usado como bactericida-fungicida, en la industria textil y como indicador en varias reacciones químicas y bioquímicas [4]. Ambos colorantes contribuyen a la contaminación de los hábitats acuáticos debido a su inapropiada disposición [5]. El CV ha sido encontrado en el suelo y en sistemas acuáticos, se supone que es el responsable de la promoción del crecimiento de tumores en peces por lo que ha sido reportado como uno de los colorantes más tóxicos para la microbiota acuática, con una media de sobrevivencia alrededor del $21 \%$ a una concentración de $5.0 \mathrm{mg} \mathrm{L}^{-1}[5,6]$. También se ha encontrado que ambos colorantes son cancerígenos y poseen efecto mutagénico [7-9]. Para disminuir la contaminación que provocan los colorantes se han buscado alternativas biológicas, dentro de las cuales se encuentra el uso de hongos filamentosos, y entre los más utilizados en sistemas in vitro están Pleurotus ostreatus, Phanerochaete chrysosporium, Rhizopus arrhizus, Penicillium restrictum y Cuninghamella elegans [10-14]. Cabe mencionar que los hongos filamentosos emplean la biosorción, biodegradación y bioacumulación para la decoloración de los colorantes [15]. El proceso de biosorción consiste en la captación pasiva del colorante en su biomasa (viva o muerta), basado en la propiedad que posee la biomasa para enlazar y acumular diferentes tipos de colorantes, este proceso ha ganado una atención considerable en las últimas décadas como un método alternativo y menos costoso para eliminar colorantes de efluentes textiles, debido a que los actuales procesos de adsorción resultan ser costosos y presentar problemas de regeneración $[16,17]$. Considerando lo anterior el presente trabajo determinó la capacidad de biosorción de dos especies del género Trichoderma (hongos ampliamente estudiados para el control biológico) frente a CV y AT, y comparó su biosorción con la adsorción que presenta el carbón activado frente a estos dos colorantes. La importancia de realizar este estudio radica en generar información adicional a la ya reportada, puesto los estudios de biosorción y degradación de colorantes empleando especies del género Trichoderma se enfocan principalmente a las especies $T$. harzianum y $T$. $s p$., dentro de estos estudios se encuentra la remoción de rodamina $6 \mathrm{G}$, azul de tripano y erioglaucina por el micelio de $T$. harzianum y la decoloración de rodamina $6 \mathrm{G}$, azul de tripano y erioglaucina por la enzima lacasa proveniente de $T$. harzianum WL1, además de la 
biosorción de naranja $\mathrm{G}$ por la biomasa de Trichoderma sp. [18-22].

\section{Parte experimental}

\subsection{Microorganismos}

Los hongos $T$. virens y $T$. viride forman parte de la colección microbiana del Laboratorio de Microbiología de Suelos del Colegio de Postgraduados, ambos fueron aislados de suelo de la rizosfera de mezquite (Prosopis sp.) en el estado de Jalisco, México [23].

\subsection{Biosorción de CV y AT por la biomasa seca de $T$. virens y $T$, viride}

Las dos cepas de Trichoderma se reactivaron en cajas de Petri con agar papa dextrosa (PDA, Merck ${ }^{\circledR}$ ) a $28{ }^{\circ} \mathrm{C}$ por 5 días. Posteriormente, se preparó una suspensión de esporas a partir de varios lavados con agua destilada estéril. Los fragmentos de micelio se removieron de la suspensión por filtración a través de fibra de vidrio estéril. La suspensión de esporas se ajustó a una concentración aproximada de $10^{6}$ esporas $\mathrm{mL}^{-1}$. A matraces Erlenmeyer de $1000 \mathrm{~mL}$ de capacidad, se les adicionaron $500 \mathrm{~mL}$ del medio mineral estéril $\left(\mathrm{g} \mathrm{L}^{-1}\right) 0.1$

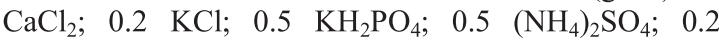
$\mathrm{MgSO}_{4} .7 \mathrm{H}_{2} \mathrm{O} ; 0.05 \mathrm{CuSO}_{4} ; 0.05 \mathrm{ZnSO}_{4} ; 0.43 \mathrm{MnSO}_{4}$; $0.05\left(\mathrm{NH}_{4}\right)_{6} \mathrm{Mo}_{7} \mathrm{O}_{24} \cdot \mathrm{H}_{2} \mathrm{O} ; 6$ glucosa y $\mathrm{pH}$ 4.3. Luego, se agregaron $10 \mathrm{~mL}$ de la suspensión de esporas $\left(10^{6}\right.$ esporas $\mathrm{mL}^{-1}$ ) de cada cepa de Trichoderma al medio de cultivo correspondiente. Los cultivos se incubaron a temperatura ambiente y se oxigenaron con una bomba de oxÍgeno por seis días. Al término de la incubación, el micelio fúngico se separó del medio de cultivo mediante filtración al vació, luego se secó a $70 \pm 2{ }^{\circ} \mathrm{C}$ por $96 \mathrm{~h}$ para determinar su peso seco y después el micelio fúngico se molió en un mortero. Los experimentos de adsorción se llevaron a cabo por la agitación de $100 \mathrm{mg}$ del respectivo adsorbente (carbón activado, biomasa seca de $T$. virens y $T$. viride) con $1 \mathrm{~mL}$ de $\mathrm{CV}$ y $\mathrm{AT}$ a las siguientes concentraciones: $0,15,30,45,60,75$ y $90 \mathrm{mg}$ $\mathrm{L}^{-1}$ respectivamente, a $25^{\circ} \mathrm{C}, \mathrm{pH}=6$ y $150 \mathrm{rpm}$ en un agitador para microtubos con termostatización (TS-100, bioSan $($ ). Debido a que los colorantes son sustancias orgánicas que presentan generalmente en su estructura química, al menos un cromóforo, sistemas conjugados y electrones de resonancia; dichas características les permite absorber luz en el espectro visible, por lo cual la espectrofotometría UV-Visible es una de las técnicas utilizada en el estudio de decoloración y adsorción de colorantes [24-29]. Considerando lo anterior, en el presente estudio la concentración de cada colorante se estimó espectrofotométricamente mediante el monitoreo de la absorbancia del CV (586 nm) y AT $(625 \mathrm{~nm})$, utilizando un lector multi-modal de microplacas (Synergy 2 SL, Biotek ${ }^{\circledR}$ ) y la capacidad de adsorción se determinó utilizando la siguiente ecuación:

$$
Q=\frac{V\left(C_{i}-C_{e}\right)}{M}
$$

Donde, $\mathrm{Q}$ es la cantidad de colorante adsorbido ( $\mathrm{mg} \mathrm{g}^{-}$ $\left.{ }^{1}\right), \mathrm{V}$ representa el volumen de la solución del colorante
(L), Ci y Ce son la concentración inicial y en equilibrio del colorante $\left(\mathrm{mg} \mathrm{L}^{-1}\right)$, y $\mathrm{M}$ es el peso seco de la biomasa fúngica $(\mathrm{g})$.

Por otra parte, considerando que durante la adsorción, se establece un rápido equilibro entre el colorante adsorbido sobre las células fúngicas muertas (qe) y el colorante no adsorbido presente en la solución (Ce), y que dicho fenómeno ha sido descrito bien a temperatura constante por los modelos de Freundlich y Langmuir en estudios anteriores [30-33]. En el presente trabajo se estudiará la capacidad de adsorción de los diferentes adsorbentes utilizando los modelos anteriormente mencionados con las siguientes ecuaciones [34,35]:

$$
q_{e}=K_{F} C_{e}^{\frac{1}{n}}
$$

Modelo Freundlich

$$
q_{e}=\frac{q_{\max } K_{L} C_{e}}{1+K_{L} C_{e}}
$$

Modelo Langmuir

Donde qmax es la capacidad máxima $\left(\mathrm{mg} \mathrm{g}^{-1}\right)$ y $\mathrm{KL}$ es el coeficiente de afinidad ( $\left.\mathrm{L} \mathrm{mg}^{-1}\right)$, mientras que qe (mg $\left.\mathrm{g}^{-1}\right)$ y Ce $\left(\mathrm{mg} \mathrm{L}^{-1}\right)$ representa la capacidad de adsorción y la concentración del colorante en la solución al equilibrio, respectivamente. $\mathrm{KF}$ y $\mathrm{n}$ representan los coeficientes de Freundlich (n, adimensional; $\mathrm{KF} \mathrm{mg}^{1-1 / \mathrm{n}}$ $\left.\mathrm{g}^{-1} 1^{1 / \mathrm{n}}\right)$.

Las cinéticas de adsorción se analizaron con los modelos de pseudo-primer y pseudo-segundo orden. El modelo de pseudo-primer orden en su forma lineal descrito por Lagergren [36]:

$$
\log \left(q_{e}-q_{t}\right)=\log \left(q_{e}\right)-\frac{K_{1}}{2.303} t
$$

Donde qe es la capacidad de adsorción en el equilibrio $\left(\mathrm{mg} \mathrm{g}^{-1}\right)$, qt es la cantidad de adsorbato adsorbido por tiempo $\mathrm{t}\left(\mathrm{mg} \mathrm{g}^{-1}\right)$ y $\mathrm{K} 1$ es la constante pseudo-primer orden $\left(\mathrm{min}^{-1}\right)$. Mientras que la forma lineal del modelo cinético pseudo-segundo orden descrito por Ho [37]:

$$
\frac{t}{q_{t}}=\frac{1}{K_{2} q_{e}^{2}}+\frac{1}{q_{e}} t
$$

Donde $\mathrm{K}_{2}$ es la constante pseudo - segundo orden ( $\mathrm{g} \mathrm{mg}$ $\left.{ }^{1} \min ^{-1}\right)$.

\section{Resultados y discusión}

\subsection{Biosorción de CV y AT por la biomasa seca de $T$. virens y $T$, viride}

Analizando los datos, se observó que la adsorción de $\mathrm{CV}$ y AT por carbón activado es rápida para ambos colorantes (Fig. 1a y 1b) y se mantuvo casi sin cambios en todos los tiempo de evaluación para el caso del CV, lo que indica que llega más rápidamente al equilibrio. En el caso de la biomasa fúngica seca de ambas especies de Trichoderma se logra apreciar mayor variabilidad en la 
adsorción de CV y AT, y no se observa si se llega a un equilibrio en los tiempos de evaluación probados (Fig. 1a y 1b). Adicionalmente el promedio de adsorción de CV por la biomasa seca de $T$. viride $(96 \%)$ y $T$. virens (94\%) no fueron tan altos como la del carbón activado (99\%) (Fig. 1a). No obstante, el promedio de adsorción para AT fue mucho menor para la biomasa seca de $T$. viride $(83 \%), T$. virens $(84 \%)$ y carbón activado $(93 \%)$ que el que tuvieron ante CV (Fig. 1b), pero también el carbón activado tuvo mayor adsorción. La tendencia de biosorción de la biomasa fúngica seca ante los dos colorantes es baja, en los primeros tiempos de evaluación tomando en consideración la adsorción del carbón activado, sin embargo, a las $24 \mathrm{~h}$ de evaluación la adsorción aumenta considerablemente.

Para este estudio se utilizaron los modelos de isotermas de Langmuir y Freundlich definidos anteriormente, y se encontró que los datos experimentales para $\mathrm{CV}$ y AT bajo las concentraciones empleadas (15-90 $\mathrm{mg} \mathrm{L}^{-1}$ ) se describen mejor mediante la isoterma de Freundlich para los tres adsorbentes (Fig. 2a y 2b). La capacidad máxima de adsorción definida como qmax (Tabla 1) muestran que Trichoderma viride presenta una mayor capacidad de adsorción para $\mathrm{CV}$ que Trichoderma virens y el carbón activado, mientras tanto para AT la especie fúngica Trichoderma virens es la que muestra mayor capacidad de adsorción. También se puede observar que la capacidad máxima de adsorción para CV es muy similar para las dos especies de Trichoderma.

Por otra parte, los datos experimentales se ajustaron mejor al modelo cinético de pseudo segundo orden (Fig. 3a y 3b) que al modelo de pseudo primer orden. El modelo de pseudo segundo orden puede explicar la cinética de adsorción de los dos colorantes en el carbón activado y la biomasa fúngica en los primeros tiempos de evaluación, ya que al incluir los datos de las evaluaciones a las 24 y $48 \mathrm{~h}$ se pierde la correlación de este modelo.

Los valores de las constantes cinéticas que se muestran en la Tabla 2, indican que es mayor la velocidad con la que se adsorbe el CV en la biomasa de $T$. virens que en los otros dos adsorbentes, mientras que para AT la velocidad de adsorción es mayor para la biomasa de $T$. viride.
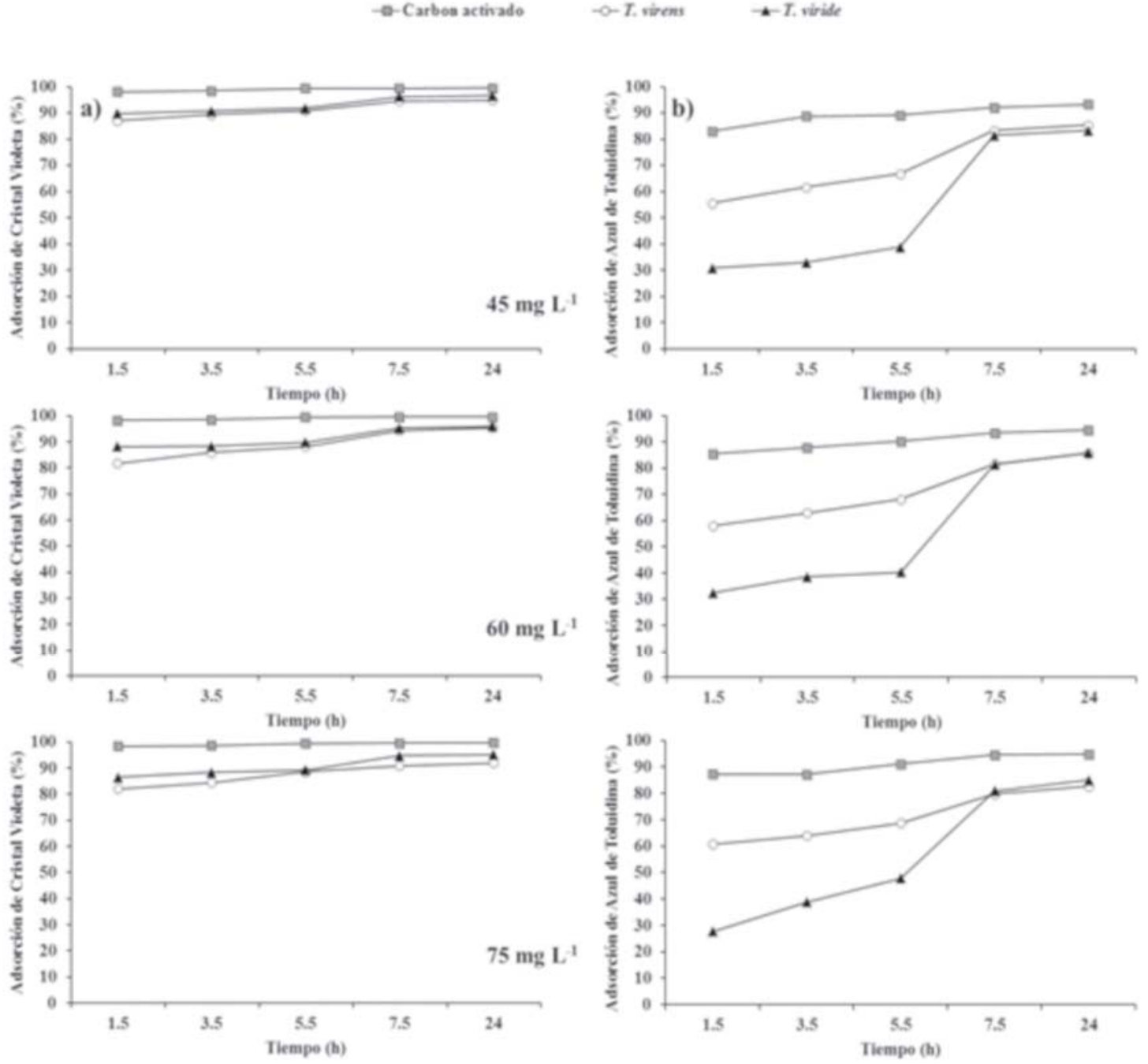

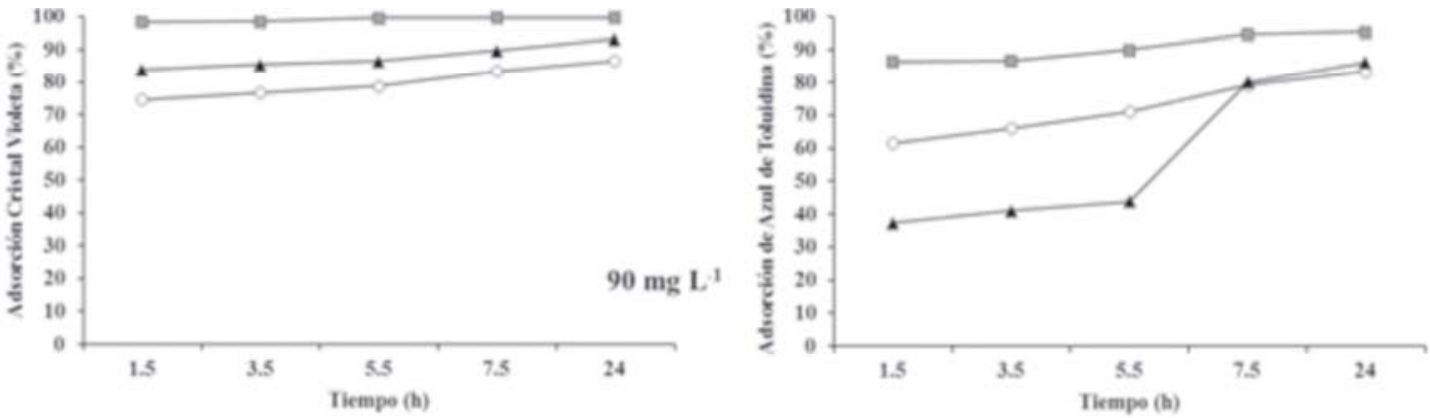

Figura1. Adsorción de dos colorantes por carbón activado y la biomasa seca de $T$. virens y $T$. viride a $25^{\circ} \mathrm{C}$ y $\mathrm{pH}=6$, a) Cristal Violeta y b) Azul de Toluidina.

Cabe mencionar que los estudios sobre la capacidad de adsorción que presentan los hongos del género Trichoderma ante colorantes como CV y AT no han sido reportados. Sin embargo, en el caso de las especies de Trichoderma se ha reportado que la biomasa fúngica viva y muerta de $T$. harzanium presentan una capacidad máxima de adsorción para el colorante azul de tripano de $4.11 \mathrm{mg} \mathrm{g}^{-1}$ y $4.13 \mathrm{mg} \mathrm{g}^{-1}$ respectivamente [19]. Mientras que la biomasa fresca de $T$. harzanium tuvo una capacidad máxima de adsorción de $3.09 \mathrm{mg} \mathrm{g}^{-1}$ para el colorante erioglaucina [20].
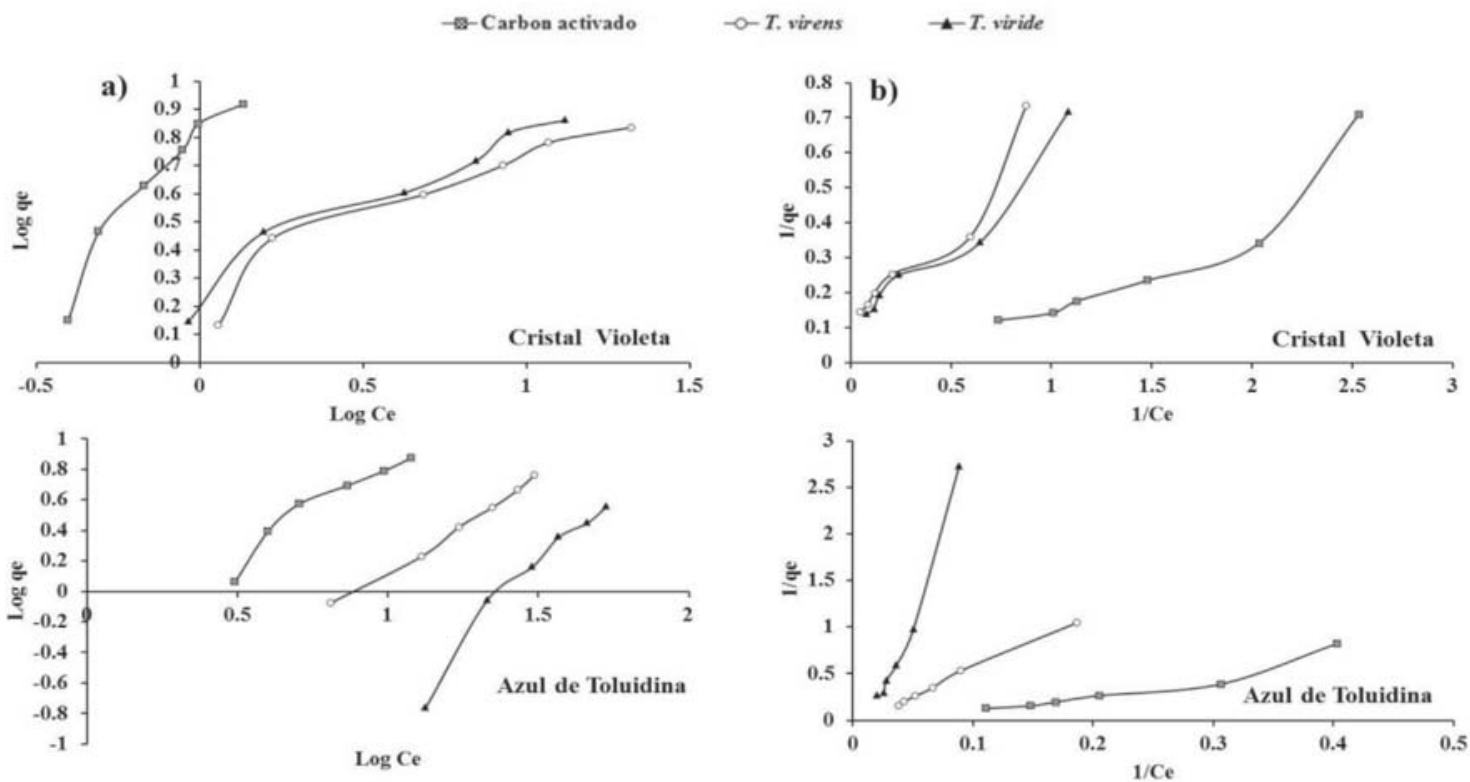

Figura 2. Isotermas de adsorción para Cristal Violeta y Azul de Toluidina por carbón activado y la biomasa seca de $T$. virens y $T$. viride a $25^{\circ} \mathrm{C}$ y $\mathrm{pH}=6$, a) Freundlich y b) Langmuir.

Tabla1. Constantes de las Isotermas de Freundlich y Langmuir para carbón activado y la biomasa seca de $T$. virens y $T$. viride ante Cristal Violeta y Azul de Toluidina (Método de mínimos cuadrados).

\begin{tabular}{|c|c|c|c|c|c|c|c|}
\hline Colorante & Adsorbente & $\begin{array}{l}\text { Freundlich } \\
\mathrm{KF}\end{array}$ & $\mathrm{n}$ & $\mathrm{R}^{2}$ & $\begin{array}{l}\text { Langmuir } \\
\text { qmax }\end{array}$ & KL & $\mathrm{R}^{2}$ \\
\hline \multirow[t]{3}{*}{$\mathrm{CV}$} & Carbón activado & 6.479 & 0.730 & 0.929 & 6.053 & 0.542 & 0.878 \\
\hline & T.virens & 2.997 & 2.319 & 0.912 & 9.293 & 0.171 & 0.912 \\
\hline & T. viride & 3.126 & 1.699 & 0.945 & 10.395 & 0.181 & 0.948 \\
\hline \multirow[t]{3}{*}{ AT } & Carbón activado & 0.407 & 0.713 & 0.960 & 5.518 & 0.080 & 0.919 \\
\hline & T.virens & 0.125 & 0.855 & 0.988 & 21.691 & 0.093 & 0.995 \\
\hline & T. viride & 0.104 & 0.658 & 0.984 & 1.578 & 0.017 & 0.975 \\
\hline
\end{tabular}

Tool, Norway: DNV, 1999. 

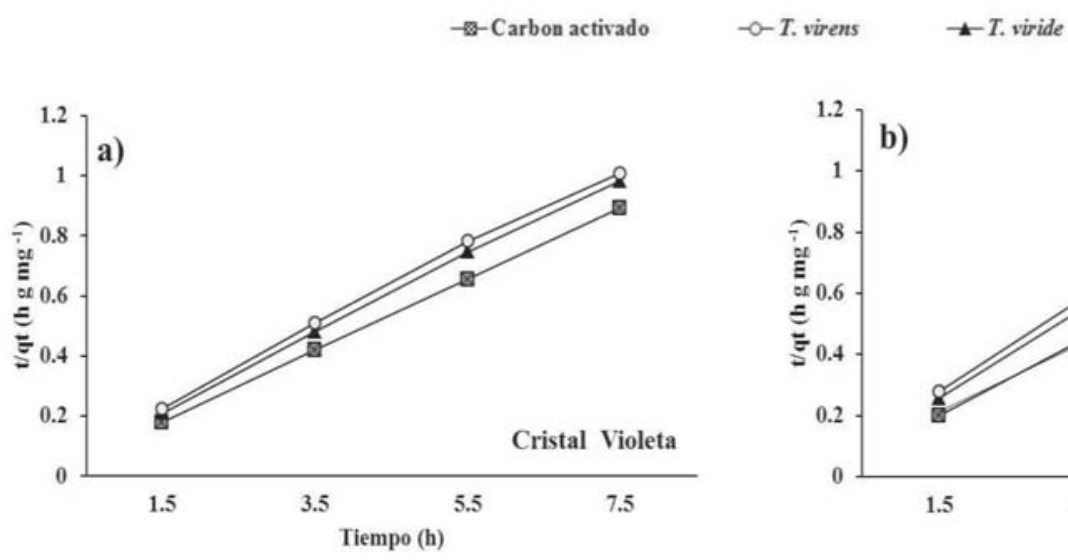

Figura 3. Modelo cinético de pseudo segundo orden para carbón activado y la biomasa seca de T. virens y $T$. viride a $25^{\circ} \mathrm{C}$ y $\mathrm{pH}=6$, a) Cristal Violeta y b) Azul de toluidina.

Por otra parte, con respecto al colorante $\mathrm{CV}$ se ha reportado que el hongo basidiomiceto Ceriporia lacerata P2 presenta una adsorción máxima de 239.25 $\mathrm{mg} \mathrm{g}^{-1}$ la cual es bastante alta en comparación con las obtenidas para $T$. viride $\left(10.39 \mathrm{mg} \mathrm{g}^{-1}\right)$ y $T$. virens $(9.29$ $\mathrm{mg} \mathrm{g}^{-1}$ ) en el presente trabajo, pero a la vez la adsorción máxima de las dos especies de Trichoderma es 2.50 y 2.24 mayor que la que se reporta para la biomasa de la hoja de Calotropis procera $\left(4.14 \mathrm{mg} \mathrm{g}^{-1}\right)$ ante $\mathrm{CV}$ $[38,39]$.

Tabla 2. Constantes del modelo cinético de pseudo segundo orden para carbón activado y la biomasa seca de $T$. virens y $T$. viride a $25^{\circ} \mathrm{C}$ y $\mathrm{pH}=6$, ante $\mathrm{CV}$ y $\mathrm{AT}$.

\begin{tabular}{lllll}
\hline Colorante & Adsorbente & $\mathrm{qe}$ & $\mathrm{K} 2$ & $\mathrm{R}^{2}$ \\
\hline $\mathrm{CV}$ & $\begin{array}{l}\text { Carbón } \\
\text { activado }\end{array}$ & 4.217 & 1.027 & 1.000 \\
& $\begin{array}{l}\text { T.virens } \\
\text { T. viride }\end{array}$ & 3.815 & 2.935 & 0.997 \\
$\mathrm{AT}$ & Carbón & 4.871 & 1.659 & 0.999 \\
& activado & & 2.323 & 0.997 \\
& T.virens & 3.732 & 1.785 & 0.998 \\
& T. viride & 4.379 & 3.878 & 0.993 \\
\hline
\end{tabular}

En el caso del colorante AT los reportes de adsorción por hongos filamentosos son aún más escasos que con $\mathrm{CV}$, no obstante se ha reportado que la adsorción máxima para la zeolita clinoptilolita es de $64.22 \mathrm{mg} \mathrm{g}^{-1}$ $\left(2.1 \times 10^{-4} \mathrm{~mol} \mathrm{~g}^{-1}\right)$ a un $\mathrm{pH}$ de 11 , la cual es mayor a la encontrada para las dos especies de Trichoderma bajo las condiciones experimentales del presente estudio, pero la adsorción máxima de $T$. virens $\left(21.69 \mathrm{mg} \mathrm{g}^{-1}\right)$ se encuentra más cercana a la reportada para el yeso gypsum que es de $28 \mathrm{mg} \mathrm{g}^{-1}[29,40]$.

\section{Conclusiones}

Aunque es evidente la falta de más investigaciones para el género de hongos Trichoderma con este tipo de enfoques, este estudio muestra que $T$. viride y $T$. virens presentan una alta remoción del colorante $\mathrm{CV}$ después de las $24 \mathrm{~h}$ de evaluación. Evidenciando el posible potencial que pueden tener las especies del género Trichoderma para el tratamiento de aguas contaminadas con este colorante.

\section{Agradecimientos}

Se agradece al CONACyT por el apoyo otorgado durante la realización del presente trabajo. Además los autores agradecen las observaciones y sugerencias al manuscrito por parte de los revisores.

\section{Referencias}

1. Zollinger, H. Color Chemistry: Syntheses, Properties, and Applications of Organic Dyes and Pigments, 3ra. ed.; Wiley-VCH: Zürich, Germany, 2003; pp 13-14.

2. Mishra, G.; Tripathi, M. Colourage. 1993, 40, 3538.

3. Azmi, W.; Sani, R. K.; Banerjee, U.C. Enzyme Microb. Tech. 1998, 22, 185-191.

4. Wainwright, M. Biotech. Histochem. 2003, 78,147-155.

5. Michaels, G. B.; Lewis, D. L. Environ. Toxicol. Chem. 1985, 4, 45-50.

6. Black, J. J.; Holmes, M.; Dymerski, P. P.; Zapisek, W.F. Fish tumor pathology and aromatic hydrocarbon pollution in a great lakes estuary. En Hydrocarbons and halogenated hydrocarbons in the aquatic environment; Afghan, B. K.; Mackay, D., Eds.; Plenum Press: New York, 1980; pp 559565.

7. Au, W.; Pathak, S.; Collie, C. J.; Hsu, T. C. Mutat. Res. 1978, 58, 269-276.

8. Dunipace, A. J.; Beaven, R.; Noblitt, T.; Li, Y.; Zunt, S.; Stookey, G. Mutat. Res. 1992, 279, $255-$ 259.

9. Chi, Z.; Liu, R.; Sun, Y.; Wang, M.; Zhang, P.; Gao, C. J. Hazard. Mater. 2010, 175, 274-278.

10. Zhao, X.; Hardin, I. R. Dyes Pigments. 2007, 73, 322-325.

11. Pazarlioglu, N. K.; Urek, R. O.; Ergun, F. Process Biochem. 2005, 40, 1923-1929.

12. Aksu, Z.; Cagatay, S. S. Sep. Purif. Technol. 2006, 48, 24-35. 
13. Cha, C.; Doerge, D. R.; Cerniglia, C. E. Appl. Environ. Microbiol. 2001, 67, 4358-4360.

14. Iscen, C.F.; Kiran, I.; Ilhan, S. J. Hazard. Mater. 2007, 143, 335-340.

15. Fu, Y.; Viraraghavan, T. Bioresour. Technol. 2001, 79, 251-262.

16. Binupriya, A. R.; Sathishkumar, M.; Swaminathan, K.; Ku, C. S.; Yun, S.E. Bioresource. Technol. 2008, 99, 1080-1088.

17. Kaushik, P.; Malik, A. Environ. Int. 2009, 35, 127-141.

18. Sadhasivam, S.; Savitha, S.; Swaminathan, K. J. Environ. Manage. 2007, 85, 155-161.

19. Sadhasivam, S.; Saritha, E.; Savitha, S.; Swaminathan, K. Bull. Environ. Contam. Toxicol. 2005, 75, 1046-1053.

20. Sadhasivam, S.; Savitha, S.; Swaminathan, K. World J. Microbiol. Biotechnol. 2007, 23, 10751081.

21. Sadhasivam, S; Savitha, S.; Swaminathan, K. World J. Microbiol. Biotechnol. 2009, 25, 17331741.

22. Sivasamy, A.; Sundarabal, N. Curr. Microbiol. 2011, 62, 351-357.

23. Ibarra-Medina, V. A.; Ferrera-Cerrato, R.; Alarcón, A.; Lara-Hernández, M. E.; ValdésCarrasco, J. M. Rev. Mex. Mic. 2010, 31, 53-63.

24. Allen, R. L. M. Colour Chemistry, Thomas Nelson and Sons Ltd: London, 1971; pp 11-13.

25. Abrahart. E. N. Dyes and their Intermediates, Chemical Publishing: New York, 1977; pp 1-12.

26. Ramsay, J. A.; Nguyen, T. Biotechnol. Lett. 2002, 24, 1757-1761.

27. Fu, Y.; Viraraghavan, T. Bioresource Technol. 2002, 82, 139-145.

28. Kumar, K. V.; Ramamurthi, V.; Sivanesan, S. Dyes Pigments. 2006, 69, 102-107.

29. Alpat, S. K.; Özbayrak, Ö., Alpat, Ş.; Akçay, H. J. Hazard. Mater. 2008, 151, 213-220.

30. Saḡ, Y., Kutsal, T. Chem. Eng. J. 1995, 181-188.

31. Gallager, K. A.; Healy, M. G.; Allen, S. J. Biosorption of synthetic dye and metal ions from aqueous effluents using fungal biomass. En Global Environmental Biotechnology; Wise, D. L, Eds; Elsevier: UK, 1997; pp 27-50.

32. Aksu, Z.; Karabayır, G. Bioresource Technol. 2008, 99, 7730-7741.

33. Aksu, Z. Process Biochem. 2005, 40, 997-1026.

34. H. M. F. J. Phys. Chem. 1906, 57, 385-470.

35. Langmuir, I. J. Am. Chem. Soc. 1916, 38, 2221 2295.

36. Lagergren, S. Kung Sven Veten Hand. 1898, 24, 1-39.

37. Ho, Y. S.; McKay, G. Process Biochem. 1999, 34, 451-465.

38. Lin, Y.; He, X.; Han, G.; Tian, Q.; Hu, W. J. Environ. Sci. 2011, 23, 2055-2062.

39. Ali, H.; Muhammad, S. K. J. Environ. Sci. Technol. 2008, 1, 143-150.

40. Rauf, M. A.; Qadri, S. M.; Ashraf, S.; AlMansoori, K. M. Chem. Eng. J. 2009, 150, 90-95. 